\title{
Association between gray matter volume in the caudate nucleus and financial extravagance: findings from voxel-based morphometry
}

Ryoichi Yokoyama, ${ }^{\mathrm{a}, \mathrm{b}}$, Takayuki Nozawa ${ }^{\mathrm{c}}$, Hikaru Takeuchi ${ }^{\mathrm{d}}$, Yasuyuki Taki ${ }^{\mathrm{de}, \mathrm{f}}$, Atsushi

Sekiguchi $^{\mathrm{a}, \mathrm{e}}$, Rui Nouchi ${ }^{\mathrm{g}}$, Yuka Kotozaki $^{\mathrm{c}}$, Seishu Nakagawa ${ }^{\mathrm{a}}$,Carlos Makoto

Miyauchi $^{\text {a,h }}$, Kunio Iizuka ${ }^{\text {a,i }}$, Takamitsu Shinada ${ }^{\text {a }}$ Yuki Yamamoto ${ }^{\text {a }}$, Sugiko Hanawa ${ }^{a}$,

Tsuyoshi Araki ${ }^{\mathrm{c}}$, Hiroshi Hashizume ${ }^{\mathrm{d}}$, Keiko Kunitoki ${ }^{\mathrm{j}}$, MayuHaninara ${ }^{\mathrm{j}}$, Yuko Sassa $^{\mathrm{d}}$, and Ryuta Kawashima ${ }^{\text {a,c,d }}$

${ }^{\mathrm{a}}$ Department of Functional Brain Imaging, Institute of Development, Aging and Cancer,

Tohoku University, Seiryo-machi 4-1, Aoba-ku Sendai, 980-8575, JAPAN

${ }^{\mathrm{b}}$ Japan Society for the Promotion of Science, Kojimachi Business Center Building,

5-3-1 Kojimachi, Chiyoda-ku, Tokyo 102-0083, JAPAN

${ }^{\mathrm{c}}$ Smart Ageing International Research Center, Institute of Development, Aging and

Cancer, Tohoku University, Seiryo-machi 4-1, Aoba-ku Sendai, 980-8575, JAPAN

${ }^{\mathrm{d}}$ Division of Developmental Cognitive Neuroscience, Institute of Development, Aging

and Cancer, Tohoku University, Seiryo-machi 4-1, Aoba-ku, Sendai 980-8575, JAPAN

${ }^{\mathrm{e}}$ Division of Medical Neuroimaging Analysis,Department of Community Medical

Supports, Tohoku Medical Megabank Organization, Tohoku University, Seiryo-machi

2-1, Aoba-ku, Sendai, 980-8573, JAPAN

Ryoichi Yokoyama

(C) 2014. This manuscript version is made available under the Elsevier user license http://www.elsevier.com/open-access/userlicense/1.0/ 
${ }^{\mathrm{f}}$ Department of Nuclear Medicine and Radiology, Institute of Development, Aging and Cancer, Tohoku University, Seiryo-machi 4-1, Aoba-ku, Sendai 980-8575, JAPAN

${ }^{g}$ Human and Social Response Research Division, International Research Institute of

Disaster Science, Tohoku University, Aoba, 6-6-4, Aoba, Sendai 980-8579 JAPAN

${ }^{\mathrm{h}}$ Graduate Schools for Law and Politics, The University of Tokyo, Hongo Bunkyo-ku

7-3-1, Tokyo 113-0033, JAPAN

i Department of Psychiatry, Tohoku University Graduate School of

Medicine, Seiryo-machi 2-1, Aoba-ku, Sendai, Miyagi, 980-8575, JAPAN

${ }^{\mathrm{j}}$ Faculty of Medicine, Tohoku University, Seiryo-machi 2-1, Aoba-ku, Sendai, Miyagi, 980-8575, JAPAN

Corresponding author:

Ryoichi Yokoyama

Department of Functional Brain Imaging, IDAC, Tohoku University

4-1 Seiryo-cho, Aoba-ku, Sendai 980-8575, Japan

Tel/Fax: +81-22-717-7988

E-mail: r-yokoyama@,idac.tohoku.ac.jp

Ryoichi Yokoyama 


\begin{abstract}
Consumer behavior differs among individuals; one such common individual difference is financial extravagance. Recent research suggests that the activation of the caudate nucleus plays an important role in consumer behavior. However, to our knowledge, no study has investigated the specific relationship between the caudate nucleus and consumer financial extravagance. Therefore, in this study, we investigated the association between individual differences in financial extravagance and regional gray matter volume in the caudate nucleus by using voxel-based morphometry (VBM). We recruited 776 healthy, right-handed individuals (432 men and 344 women; mean age $=20.7 \pm 1.8)$ and used voxel-based morphometry and a financial extravagance questionnaire to determine the association between financial extravagance and gray matter structure in the caudate nucleus. Our measure of financial extravagance was based on the novelty seeking subscales of the Japanese version of the Temperament and Character Inventory. A multiple regression analysis including financial extravagance, age, sex, Raven's Advanced Progressive Matrixscore, total intracranial volume, and novelty seeking subscales was conducted to examine what variables were related to regional gray matter volume in the region of interest within the caudate nucleus. A


significant positive correlation was found between the obtained financial extravagance score and regional gray matter volume in the caudate nucleus. We found that structural variations in the caudate nucleus contributed to individual differences in financial extravagance. This finding may provide a new neuroscientific approach to understanding individual characteristics of consumers.

Keywords: financial extravagance, voxel-based morphometry, gray matter structure, caudate nucleus.

Ryoichi Yokoyama 


\section{Introduction $^{1}$}

Consumer behavior differs among individuals; one such common individual difference is the degree of financial extravagance (i.e., excessive or unnecessary outlay)[1].

Financial extravagance is important not only in the field of psychology (e.g., it is a symptom of bipolar disorder), but also in the field of consumer behavior [2].

Recent research suggests that activation of the ventral striatum plays an important role in consumer behavior [3]. In particular, a relationship between caudate nucleus activation and compulsive buying has been established; Raab et al. found higher activation in the caudate nucleus of compulsive buyers than in that of non-compulsive buyers who viewed a product that they could buy[4]. These studies suggest that caudate nucleus activation is associated with consumer behavior.

\footnotetext{
${ }^{1} \mathrm{MRI}$, magnetic resonance imaging; rGMV, regional gray matter volume; GM, gray matter; VBM, voxel-based morphometry; TCI, Temperament and Character Inventory; NS, novelty seeking; NS1, excitability; NS2, impulsiveness; NS3, financial extravagance; NS4, disorderliness; RAPM, Raven's Advanced Progressive Matrices; SPM8, Statistical Parametric Mapping version 8; TPM, tissue probability map; T1WI, T1-weighted images; MNI, Montreal Neurological Institute
} 
Recently, through use of structural MRIs, researchers have been able to predict individual differences in a wide range of human cognitive behaviors by looking at the gray and white matter of the human brain [5]. It is possible that individual differences in caudate structure explain individual differences in financial extravagance. Indeed, previous studies have revealed that caudate dopamine synthesis capacity predicts financial extravagance in individuals with Parkinson's disease [1]. However, no study has investigated the relationship between brain structure and consumer behavior. Therefore, we devised the present study to address this gap in literature. We hypothesized, that individual differences in caudate structure form the neural basis of financial extravagance.

To test our hypothesis, we investigated the association between individual differences in financial extravagance and regional gray matter (GM) volume (rGMV) in the caudatenucleusby using voxel-based morphometry (VBM). To assess financial extravagance, we used the Japanese version [6] of the Temperament and Character Inventory (TCI)[7]. 


\section{Method}

Participants

Seven hundred and seventy-six healthy, right-handed individuals (432 men and 344

women; mean age $=20.7 \pm 1.8)$ participated in this study as part of an ongoing project

investigating associations between neural structures, cognitive functions, aging, genetics,

and daily habits [8-12]. We must note that data derived from the participants in this

study will be used in other studies unrelated to the present aims. Some of the individuals

who participated in this study were also participants of a concurrent intervention study

(psychological and imaging data that were recorded before conducting interventions,

were used in this study). Psychological tests and MRI scans not described in this study

were performed together with those described in the concurrent intervention study. All

participants had normal vision, and were undergraduate or postgraduate students, or

were individuals who had graduated within the year prior to the experiment. Participants

were recruited through advertisements on bulletin boards at Tohoku University or via

emails introducing the study. These advertisements and emails specified that individuals

with the following conditions would be unable to participate in the study: left

handedness, metal in and around the body, claustrophobia, use of certain drugs, a

history of certain psychiatric and neurological diseases, and previous participation in

Ryoichi Yokoyama 
related experiments. We provided questionnaires to all potential experimental participants for the assessment of psychiatric illnesses and recent drug use history. In the questionnaire, participants were asked to provide a detailed list of all drugs that they had recently used. None had a history of neurological or psychiatric illness. These assessments, made during recruitment and through questions after recruitment, were based on voluntary self-report. Handedness was evaluated using the Edinburgh Handedness Inventory [13]. Written informed consent was obtained from each subject in accordance with the Declaration of Helsinki (1991). This study was approved by the Ethics Committee of Tohoku University.

\section{Financial extravagance questionnaire}

Our measure of financial extravagance was based on novelty seeking (NS) as assessed by the Japanese version of the TCI. The TCI was originally developed by Cloninger et al. [7]; it was then translated into Japanese and back-translated to ensure reliability [6, 14]. The Japanese version of the TCI has good internal reliability, content validity, and construct validity in a Japanese population, and the factor structure of the inventory was consistent with Cloninger's original theory [14]. In the present study, we used the 240-item version of the TCI. Our measure of financial extravagance was one of the 
NSsubscales, termed NS3 (the other NS subscales exploratory excitability [NS1], impulsiveness [NS2], and disorderliness [NS4]). NS3 is widely used to measure individual differences in the tendency towards financial extravagance [1, 15].

Psychometric measures of general intelligence

We used Raven's Advanced Progressive Matrix (RAPM) [16] to assess intelligence, because it is regarded as one of the purest psychometric measures of general intelligence [16], and has perhaps the strongest correlation with the actual construct. We used this test to adjust for the effects of individual psychometric measures of intelligence on brain structures [17-19]. RAPM contains 36 nonverbal items requiring fluid reasoning ability. Each item consists of a $3 \times 3$ matrix with a missing piece to be completed by selecting the best of eight alternatives[16]. This test score (number of correct answers in 30 minutes) was used as an index of individual psychometric measures of intelligence.

\section{Behavioral data analysis}

To confirm the relationship between financial extravagance score (NS3) and other psychological variables, we conducted multiple regression analysis using the following 
model:

$\mathrm{NS} 3=\beta 0+\beta 1(\mathrm{Age})+\beta 2(\mathrm{Sex})+\beta 3(\mathrm{RAPM})+\beta 4(\mathrm{NS} 1)+\beta 5(\mathrm{NS} 2)+\beta 6(\mathrm{NS} 4)+$

$\omega$, where NS3 denotes the financial extravagance score; $\beta 0$ denotes the intercept;Age,

Sex, and RAPM denotes age, sex, and RAPM score, respectively; NS1, NS2, and NS4

denotes exploratory excitability, impulsiveness, and disorderliness, respectively; and $\omega$

denotes the residual.

Image acquisition and analysis

All MRI data was acquired using a 3-T Philips Achieva scanner. High-resolution

T1-weighted structural images (T1WIs: $240 \times 240$ matrix, TR $=6.5 \mathrm{~ms}, \mathrm{TE}=3 \mathrm{~ms}$,

FOV $=24 \mathrm{~cm}$, slices $=162$, slice thickness $=1.0 \mathrm{~mm}$ ) were collected using a

magnetization-prepared rapid gradient echo sequence.

Preprocessing of T1-weighted structural data

Preprocessing of the structural data was performed using Statistical Parametric Mapping

software (SPM8; Wellcome Department of Cognitive Neurology, London, UK)

implemented in Matlab (Mathworks Inc., Natick, MA, USA). Using the new

segmentation algorithm implemented in SPM8, T1WIs of each individual were

Ryoichi Yokoyama 
segmented into six tissues. In this process, we modified the original SPM8 gray matter tissue probability map (TPM) by first reducing the signal intensities of the voxels (gray matter tissue probability of the default tissue gray matter TPM + white matter tissue probability of the default TPM) that are greater than 0.25 to 0 . When this modified gray matter TPM is used, the dura matter is less likely to be classified as gray matter (compared with when the default gray matter TPM is used), without other substantial segmentation problems. In this new segmentation process, we used default parameters; however, affine regularization was performed with the International Consortium for Brain Mapping template for East Asian brains. We then proceeded to the diffeomorphic anatomical registration through an exponentiated lie algebra registration process(DARTEL), implemented in SPM8. In this process, we used DARTEL import images of the five TPMs from the abovementioned new segmentation process. First, for the DARTEL procedures, the template we created for our previous study [20] was prepared (this template was also used in a different previous study [21]). Next, using this existing template, the DARTEL procedures were performed for all of the participants in the present study. In these procedures, default parameter settings were used. The resulting images were spatially normalized to the standards of the Montreal Neurological Institute (MNI), giving images with $1.5 \times 1.5 \times 1.5 \mathrm{~mm}^{3}$ voxels. 
Subsequently, all images were smoothed by convolving them with an isotropic Gaussian kernel of $4 \mathrm{~mm}$ full width at half maximum (FWHM).

\section{Definition of ROI}

In this study, we predicted that individual differences in the caudate nucleus form the neural basis for individual differences in financial extravagance. Therefore, we defined the region of interest (ROI) within the caudatenucleus. The ROI was defined by the Wake Forest University PickAtlas (www.fmri.wfubmc.edu) with the AAL ROI package.[22].

\section{Statistical analyses}

We investigated rGMV associated with financial extravagance. Statistical analyses of morphological data were then performed using VBM8 software, an extension of SPM8. In the analyses, we included only voxels that showed rGMV values greater than 0.05 in all subjects. The primary purpose for using GM thresholds was to cut the periphery of the GM areas so that the areas for analysis were effectively limited.

Given our a priori hypothesis on the role of the caudate nucleusin consumer behavior, 
we focused on the caudatenucleus (specifically, the ROI defined above). Using multiple regression analysis, we tested for a relationship between financial extravagance (as assessed by the NS3 subscale of the TCI) and rGMV. Analyses were performed using sex, age, RAPM score, and total intracranial volume (TIV; total GM volume + total $\mathrm{WM}$ volume + total CSF volume) as additional covariates. Furthermore, other NS subscales such as NS1, NS2, and NS4 were also included as additional covariates to control for the effect of these factors, resulting in a total of eight covariates. Total brain volume was included as a covariate in some of the analyses of volume measures in order to assess the volumes of tissues that cannot be assessed using total brain volume alone. The statistical significance level was set at $P<0.05$ for our investigation of the ROI defined above, with a small volume correction for multiple comparisons (voxel-level family-wise error).

As an additional analysis, the whole-brain multiple regression analysis was also conducted to clarify if there were any regions significantly correlated to financial extravagance outside of our ROI. The same procedure as above was employed for this analysis. The statistical significance level in this study was set at $P<0.05$ for multiple comparisons(voxel-level family-wise error) for the whole-brain analysis. 


\section{Results}

Basic data

Table 1 shows the means and standard deviations (SD) of participants' age, RAPM

scores, and financial extravagance scores. The mean score in our sample for NS3

(financial extravagance) was $5.28 \pm 1.97$. ThisNS3 score was comparable to that of

age-matched healthy male controls in a previous study $(n=851$; mean age $22.1 \pm 2.84$;

$\mathrm{NS} 3=4.8 \pm 2.2)[23]$

A multiple regression analysis - with the financial extravagance score as the dependent

variable, and age, sex, and RAPM score as independent variables - revealed that age

had a significant positive effect on financial extravagance. Other NSsubscales also had

significant positive effects on financial extravagance (Table 2).

ROI analysis (small volume correction): Correlation between rGMV in the caudate

nucleus and financial extravagance

We investigated the association between rGMV in the caudate nucleus and individual

Ryoichi Yokoyama 
differences in financial extravagance in the ROI. A multiple regression analysis

including age, sex, RAPM score, TIV, and the other three NS subscales as covariates revealed a significant positive correlation between financial extravagance score and

rGMV in the caudate nucleus (MNI: 17, 3, 13, $t=4.01, P<0.029$ [family-wise error collected using small volume correction in the ROI]) (Table 3). We also conducted an identical analysis using other NS subscales (NS1,NS2 and NS4); however, we did not find any specific region that significantly related to these subscales.

Whole-brain analysis: Correlation between rGMV and financial extravagance

We also investigated the association between whole-brain rGMV and individual differences in financial extravagance, using the same multiple regression analysis procedure as stated above. The whole-brain analysis did not reveal any specific region significantly related to financial extravagance (NS3 scores;Table 3). This was similarly true for the other TCI subscales (NS1, NS2, and NS4).

\section{Discussion}

To the best of our knowledge, this is the first study to investigate associations between 15

Ryoichi Yokoyama 
brain structures and financial extravagance. The results indicated that greater rGMV in the caudatenucleus is associated higher financial extravagance. This supports our hypothesis that the caudate nucleus forms part of the neural basis of financial extravagance.

A previous study showed that gray matter volume in the caudate nucleus positively correlated with measures of obesity [24]. This study indicated a relationship between reward sensitivity, particularly the relationship between the reward obtained from eating, and the caudatenucleus, explained as a tendency of higher reward sensitivity for food among individuals with obesity. Thus, reward sensitivity is linked to caudate volume and function. Since financial extravagance could be related to the sensitivity of reward obtained from shopping, our finding that caudate rGMV is associated with financial extravagance is consistent with findings from previous studies.

Furthermore, a previous study suggested that ventral striatal (including the caudate nucleus) dopamine synthesis capacity is related to financial extravagance[1],which provide the biological underpinnings of variation in rGMV in the caudatenucleus. In other words, the individual differences in dopamine synthesis capacity in the caudate 
nucleus may correspond to individual differences in caudate volume observed in our study - thus, a high rGMV in the caudate nucleus could be related to high dopamine synthesis capacity in that area, resulting in the tendency toward greater financial extravagance.

Previous studies have reported that the caudate nucleus is also associated with impulsivity [25]. Furthermore, in this study, at the behavioral level, financial extravagance (NS3) has been suggested to have a high correlation with impulsiveness (NS2) [26]. Indeed, the results of our behavioral analysis replicated the high correlation between these measurements (Table 2). Importantly, we included other NS subscales as additional covariates to clarify whether financial extravagance was related to caudate volume per se. Despite our inclusion of other NS variables, weobserved a singular definitive relationship between caudate volume and financial extravagance. This result is consistent with that of a previous study[1] that found thatdespite no significant correlation between impulsiveness (NS2) and ventral striatal dopamine synthesis capacity, there was a significant correlation between financial extravagance (NS3) and ventral striatal dopamine synthesis capacity. 
A previous study reported that there is laterality regarding functions in the caudate nucleus[27]. However, we found bilateral activation in the caudatenucleus by using a more liberal threshold for significance $(P<0.005)$, uncorrected at the voxel level (Fig. 1.). Thus, laterality is inconsequential for our results.

The results of our behavioral study also suggested that age has a positive effect on financial extravagance. Age may be related to an individual's economic situation. Our participants were university students ranging from first-year students to recent graduates. Thus, we anticipated a substantial income difference among them, with older students earning more, and parental control over finances gradually reducing as individuals progress through young adulthood. Therefore, older students might spend their money more freely and consequently tended towards greater financial extravagance. However, we adjusted for the effect of age in this study, and found that age differences did not affect our main results.

It was of interest to investigate the other NS scores and their neural correlates.

Therefore, we conducted additional analyses using an identical procedure for NS1, NS2, andNS4. However, no brain regions were identified as significant from these analyses. 
This study has some limitations. First, since we did not perform official, in-depth diagnostic procedures for all possible existing diseases, it was possible that some participants could have had undiagnosed medical conditions; thus, our results may be somewhat biased. Second, high alcohol use could be a potential confounding factor [28] that we could not control for, since we did not collect alcohol consumption data from our participants. Third, although the reliability (i.e., internal consistency, inter-rater)of the TCI has been confirmed, and a previous study used the same subscale of the TCI as an indicator of extravagance, the test-retest reliability of NS3was not checked in this study.

Our findings have significant implications for consumer behavior research and consumer neurosciencesince it introduces the possibility of characterizing consumers by their brain structures. This approach could lead to new methods of understanding individual characteristics of consumers from a neuroscientific perspective. 


\section{Acknowledgments}

We would like to thank Yuki Yamada for operating the MRI scanner, Haruka Nouchi for

conducting the psychological tests, and all our colleagues at the Institute of

Development, Aging, and Cancer.

This study was supported by JST/RISTEX, JST/CREST, a Grant-in-Aid for Young

Scientists (B) (KAKENHI 23700306), and a Grant-in-Aid for Young Scientists (A)

(KAKENHI 25700012) from the Ministry of Education, Culture, Sports, Science and

Technology, the Japan Society for the Promotion of Science, and Tohoku University

International Advanced Research and Education Organization.

\section{References}

[1] A.D. Lawrence, D.J. Brooks, A.L. Whone, Ventral striatal dopamine synthesis capacity predicts financial extravagance in Parkinson's disease, Frontiers in psychology 4 (2013) 90.

[2] B. Verplanken, A. Herabadi, Individual differences in impulse buying tendency: Feeling and no thinking, Eur J Personality 15 (2001) S71-S83.

[3] B. Knutson, S. Rick, G.E. Wirnmer, D. Prelec, G. Loewenstein, Neural predictors of purchases, Neuron 53 (2007) 147-156.

[4] G. Raab, C. Elger, M. Neuner, B. Weber, The neural basis of compulsive buying, Compulsive buying. Clinical foundations and treatment.. New York: Routledge (2010) 63-86.

[5] R. Kanai, G. Rees, The structural basis of inter-individual differences in human behaviour and cognition, Nat Rev Neurosci 12 (2011) 231-242.

[6] N. Kijima, R. Saito, M. Takeuchi, A. Yoshino, Y. Ono, M. Kato, T. Kitamura, Cloninger's seven-factor model of temperament and character and Japanese version of Temperament and Character Inventory (TCI), Archives of Psychiatric Diagnosis 
and Clinical Evaluation 7 (1996) 379-399.

[7] C.R. Cloninger, D.M. Svrakic, T.R. Przybeck, A psychobiological model of temperament and character, Archives of General Psychiatry 50 (1993) 975-990.

[8] H. Takeuchi, Y. Taki, H. Hashizume, Y. Sassa, T. Nagase, R. Nouchi, R. Kawashima, Failing to deactivate: the association between brain activity during a working memory task and creativity., Neuroimage 55 (2011) 681-687.

[9] H. Takeuchi, Y. Taki, Y. Sassa, H. Hashizume, A. Sekiguchi, A. Fukushima, R. Kawashima, Regional gray matter density associated with emotional intelligence: evidence from voxel-based morphometry, Human Brain Mapping (in press).

[10] Y. Taki, H. Hashizume, Y. Sassa, H. Takeuchi, M. Asano, K. Asano, Y. Kotozaki, R. Nouchi, K. Wu, H. Fukuda, Correlation among body height, intelligence, and brain gray matter volume in healthy children, Neuroimage 59 (2011) 1023-1027.

[11] H. Takeuchi, Y. Taki, R. Nouchi, A. Sekiguchi, Y. Kotozaki, C.M. Miyauchi, R. Yokoyama, K. Iizuka, H. Hashizume, S. Nakagawa, A voxel-based morphometry study of gray and white matter correlates of a need for uniqueness, Neuroimage 63 (2012) 1119-1126.

[12] H. Takeuchi, Y. Taki, R. Nouchi, H. Hashizume, A. Sekiguchi, Y. Kotozaki, S. Nakagawa, C.M. Miyauchi, Y. Sassa, R. Kawashima, Anatomical correlates of self-handicapping tendency, Cortex 49 (2013) 1148-1154.

[13] R.C. Oldfield, The Assessment and Analysis of Handedness: The Edinburgh Inventory, Neuropsychologia 9 (1971) 97-113.

[14] N. Kijima, E. Tanaka, N. Suzuki, H. Higuchi, T. Kitamura, Reliability and validity of the Japanese version of the Temperament and Character Inventory, Psychol Rep 86 (2000) 1050-1058.

[15] N. Bernow, B. Kruck, P. Pfeifer, K. Lieb, O. Tuscher, C. Fehr, Impulsiveness and Venturesomeness in German Smokers, Nicotine Tob Res 13 (2011) 714-721.

[16] J. Raven, Manual for Raven's progressive matrices and vocabulary scales, Oxford Psychologists Press, Oxford, 1998.

[17] H. Takeuchi, Y. Taki, Y. Sassa, H. Hashizume, A. Sekiguchi, A. Fukushima, R. Kawashima, White matter structures associated with creativity: Evidence from diffusion tensor imaging, Neuroimage 51 (2010) 11-18.

[18] H. Takeuchi, Y. Taki, Y. Sassa, H. Hashizume, A. Sekiguchi, A. Fukushima, R. Kawashima, Regional gray matter volume of dopaminergic system associate with creativity: Evidence from voxel-based morphometry Neuroimage 51 (2010) 578-585.

[19] R.J. Haier, R.E. Jung, R.A. Yeo, K. Head, M.T. Alkire, Structural brain variation and 
general intelligence, Neuroimage 23 (2004) 425-433.

[20] H. Takeuchi, Y. Taki, H. Hashizume, Y. Sassa, T. Nagase, R. Nouchi, R. Kawashima, Failing to deactivate: the association between brain activity during a working memory task and creativity, Neuroimage 55 (2011) 681-687.

[21] H. Takeuchi, Y. Taki, B. Thyreau, Y. Sassa, H. Hashizume, A. Sekiguchi, T. Nagase, R. Nouchi, A. Fukushima, R. Kawashima, White matter structures associated with empathizing and systemizing in young adults, Neuroimage 77 (2013) 222-236.

[22] N. Tzourio-Mazoyer, B. Landeau, D. Papathanassiou, F. Crivello, O. Etard, N. Delcroix, B. Mazoyer, M. Joliot, Automated anatomical labeling of activations in SPM using a macroscopic anatomical parcellation of the MNI MRI single-subject brain, Neuroimage 15 (2002) 273-289.

[23] S.M. Sung, J.H. Kim, E. Yang, K.Y. Abrams, I.K. Lyoo, Reliability and validity of the Korean version of the Temperament and Character Inventory, Compr Psychiat 43 (2002) 235-243.

[24] Y. Taki, S. Kinomura, K. Sato, K. Inoue, R. Goto, K. Okada, S. Uchida, R. Kawashima, H. Fukuda, Relationship between body mass index and gray matter volume in 1,428 healthy individuals, Obesity 16 (2008) 119-124.

[25] S.M. Brown, S.B. Manuck, J.D. Flory, A.R. Hariri, Neural basis of individual differences in impulsivity: Contributions of corticolimbic circuits for behavioral arousal and control, Emotion 6 (2006) 239-245.

[26] J.D. Flory, P.D. Harvey, V. Mitropoulou, A.S. New, J.M. Silverman, L.J. Siever, S.B. Manuck, Dispositional impulsivity in normal and abnormal samples, J Psychiat Res 40 (2006) 438-447.

[27] G.W. Schrimsher, R.L. Billingsley, E.F. Jackson, B.D. Moore, Caudate nucleus volume asymmetry predicts attention-deficit hyperactivity disorder (ADHD) symptomatology in children, J Child Neurol 17 (2002) 877-884.

[28] T.L. Jernigan, N. Butters, G. Ditraglia, K. Schafer, T. Smith, M. Riwin, I. Grant, M. Schuckit, L.S. Cermak, Reduced Cerebral Gray-Matter Observed in Alcoholics Using Magnetic-Resonance-Imaging, Alcohol Clin Exp Res 15 (1991) 418-427. 
Tables

Table 1 Demographics of participants.

\begin{tabular}{lcc}
\hline Measure & Mean & SD \\
\hline Age & 20.70 & 1.84 \\
RAPM & 28.63 & 3.73 \\
Financial extravagance (NS3) & 5.28 & 1.97 \\
\hline
\end{tabular}

Note. RAPM = Raven's Advanced Progressive Matrices 
Table 2. Statistical values of multiple regression analyses between the financial extravagance score (NS3) and other psychological variables

\begin{tabular}{llll}
\hline Variables & $P$ & $t$ & $\beta$ \\
\hline Age & $<0.001$ & 3.39 & 0.12 \\
Sex $($ Female $=1$, Male $=0)$ & 0.24 & -1.18 & -0.16 \\
RAPM & 0.30 & 1.04 & 0.02 \\
NS1 & $<0.001$ & 5.17 & 0.15 \\
NS2 & $<0.001$ & 7.10 & 0.23 \\
NS4 & 0.01 & 2.46 & 0.09 \\
& & & -0.13 \\
\hline Intercept) & 0.89 & -0.14 & \\
\hline
\end{tabular}

Note. NS1 = excitability, NS2 = impulsiveness, NS4 = disorderliness, RAPM = Raven's Advanced Progressive Matrices 
Table 3Correlation between financial extravagance and rGMV.

\begin{tabular}{lllllll}
\hline \hline & \multicolumn{5}{c}{ MNI peak coordinates $(\mathrm{mm})$} & \\
\cline { 2 - 4 } Area & $\mathrm{x}$ & $\mathrm{y}$ & $\mathrm{z}$ & & $\mathrm{k}$ \\
\hline $\begin{array}{l}\text { Small volume correction in caudate } \\
\text { Caudate L }\end{array}$ & 17 & 3 & 13 & 4.01 & 18 \\
\hline
\end{tabular}

Local correlation between financial extravagance and rGMV are presented as Montreal Neurological Institute coordinates (cluster peak expressed as the $t$ value). " $\mathrm{k}$ " indicates the cluster size. The statistical significance level was set at $P<0.05$ for our investigation of the ROI, with a small volume correction for multiple comparisons (voxel-level family-wise error). 
Figures
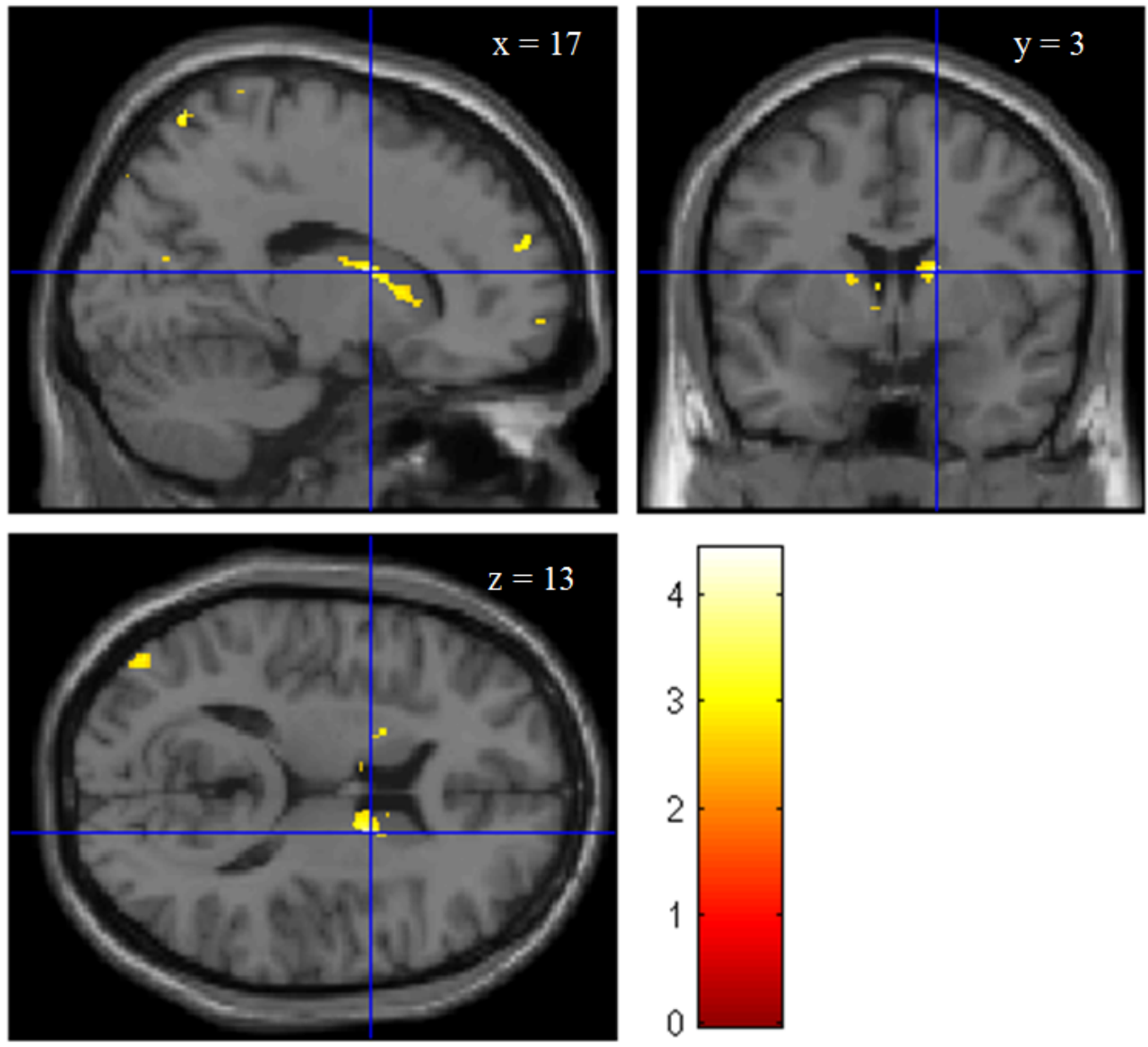

Fig. 1. Anatomical correlates of financial extravagance.

The region of correlation is overlaid on a sagittal section (top left), a coronal section (top right), and an axial section (bottom left) of the T1-weighted anatomical images provided by SPM8. The red-yellow color scale indicates the $t$ score of the positive correlation between regional gray matter volume (rGMV) and financial extravagance score. Results are shown with an underlying voxel peak level of $P<0.005$ for 
visualization purposes.

Ryoichi Yokoyama 